\title{
The Mobilities of Carriers Confined in a Single-side Doped Square Quantum Wells Dependence on Temperature
}

\author{
Tran Thi Hai ${ }^{*}$ \\ Faculty of Nature Science, Hong Duc University, Thanh Hoa, Vietnam
}

Received 17 April 2017

Revised 26 May 2017; Accepted 15 June 2017

\begin{abstract}
A theory is given of the mobility of a two-dimensional electron gas at high temperature in single-side square quantum wells. Within the variational approach, we obtain analytic expressions for the carrier distribution, and autocorrelation functions for various scattering mechanisms. We examine the dependence of the mobilities of carriers on the temperature. Our theory is able to well reproduce the recent experimental data on transport in 1S-doped square QWs, e.g., acoustic-phonon partial mobility dependence on temperature for single-side modulation doped square quantum wells.

Keywords: Single-side (1S) doing, variational approach, mobility, square quantum wells.
\end{abstract}

\section{Introduction}

Modulation doped quantum wells (QWs) have received enormous attention in recent years due to their importance in device applications. In order to upgrade the electron mobility of modulation doped quantum wells, one needs to identify the key scattering mechanisms limiting the transport properties of their two-dimensional electron gas (2DEG). It is well known $[1,2]$ that the best way for this purpose is to study the dependence of 2DEG mobility on experimental conditions such as sample temperature, carrier density, and channel width.

Recently, $[3,4]$ we have presented a first successful attempt at giving a theory of the mobilities of carriers confined in a single-side doped square quantum. We are able to explain the mobility of the single-side doped square quantum well dependence on carrier density and channel width. However, these investigation do not take into account mobility dependence on temperature. Thus, the goal of this paper is to provide a theory of the band bending effect on the high-temperature transport properties of charge carriers in a single-side modulation doped infinite square QW. We develop a variational approach to the description of quantum confinement in bent-band infinite square QWs. For strained GaAs channel in GaAs/ AlGaAs single asymmetric quantum well, we incorporate all possible scattering mechanisms, especially optical phonons (dominant at high temperatures).

\footnotetext{
* Tel.: 84- 915017980 .

Email: tranthihai@hdu.edu.vn

https://doi.org/10.25073/2588-1124/vnumap.4207
} 
The paper is organised as follows. In the second section, we supply the basic equations for calculating the carrier distribution in the growth direction for single-side doped square QWs. In section 3, Numerical results and comparison with the recent experimental data. Lastly, a summary is given in section 4 .

\section{Basic relations}

\subsection{Variational wave function for a single-side modulation doped infinite square $Q W$}

The well layer forms a conduction channel of width $L$ in the region $|z|<L / 2$. The sample is modulation doped in a region of thickness $L_{d}$ in the surface-side barrier $z<-L / 2$ and separated from the channel by a spacer layer of thickness $L_{s}$. The confinement may be plausibly described by an asymmetric wave function as follows [3]:

$$
\zeta(z)=\left\{\begin{array}{lr}
B k^{1 / 2}(\cos k z) e^{-\kappa z} & \text { for }|z|<L / 2 \\
0 & \text { for }|z|>L / 2,
\end{array}\right.
$$

in which $B, k$ and ${ }^{\kappa}$ are variational parameters to be determined; $k$ and ${ }^{\kappa}$ are wave numbers in the well layer with ${ }^{K}$ quantifying the doping - induced band bending effect. The boundary conditions at the interface planes and the normalization read as follows:

$$
k=\pi / L, \pi B^{2} \gamma_{1}(c) / 2=1,
$$

where $c=\kappa L$, and $\gamma_{1}(c)$ is in Eq.(18), so $c$ is the single independent variational parameter. In the absence of doping $c=0$ this goes to the exact symmetric wave function of an ideally-square well (flatband model). [5] Thus, the parameter $c$ quantifies the effect of the doping-induced band bending on the wave function.

\subsection{Hartree potential in a single-side modulation doped infinite square $Q W$}

The wave function of the lowest subband is to minimize the total energy per particle, which is determined by the Hamiltonian

$$
H=T+V_{H}(z),
$$

where $T$ is the kinetic energy, and $V_{H}(z)$ is the Hartree potential as a confining potential along the growth direction. The latter is created by the ionized remote impurities and charge carriers in the well in accordance with Poisson's equation:

$$
\frac{d^{2}}{d z^{2}} V_{H}(z)=\frac{4 \pi e^{2}}{\varepsilon_{L}}\left[N_{I}(z)-p(z)\right]
$$

in which $N_{I}(z)$ is the bulk density of impurities (per unit volume), $p(z)$ the one of carriers, and $\varepsilon_{L}$ is the dielectric constant of the QW. The sample is modulation doped with an impurity density $N_{I}$ in a region in the top barrier from $-z_{d}$ to $-z_{s}$, where the doping positions: $z_{d}=L_{d}+L_{s}+L / 2$ and $z_{s}=L_{s}+$ $L / 2$, so that

$$
N_{I}(z)=\left\{\begin{array}{l}
N_{I}, \text { for }-z_{d} \leq z \leq-z_{s} \\
0, \quad \text { elsewhere, }
\end{array} \text { and } p(z)=p_{s}|\zeta(z)|^{2},\right.
$$


with $p_{s}$ as a sheet carrier density. We solve Poisson's equation (4) in combination with electrostatic boundary conditions, especially the vanishing of the relevant electric field at infinity $z \rightarrow \pm \infty$. As a result, within the variational approximation with the use of the wave function from Eq.(1), the Hartree potential may be separated into two parts:

$$
V_{H}(z)=V_{I}(z)+V_{s}(z) \text {. }
$$

The first term $V_{I}$ from Eq.(6) is to be regarded as the impurity potential fixed by the doping profile, viz the impurity density $N_{I}$ and doping positions $z_{d}$, $z_{s}$; while the second one $V_{s}$ as the carrier potential fixed by their sheet density $p_{s}$ and their distribution.

\subsection{Total energy per particle in the lowest subband}

We now turn to the total energy per particle in the ground-state subband. The expectation value for the Hamiltonian from Eqs.(3) and (6) is a function of the bending parameter $c$, given by

$$
E(c)=\langle T\rangle+\left\langle V_{I}\right\rangle+\left\langle V_{s}\right\rangle \text {. }
$$

The total energy per particle is given by Eq.(7), in which the average potential due to the carrier distribution is to be replaced with its half. For the kinetic energy, it holds

$$
\langle T\rangle=\frac{-\hbar^{2}}{2 m_{z} L^{2}} \frac{\pi B^{2}}{2}\left[\left(c^{2}-\pi^{2}\right) \gamma_{1}(c)+2 \pi c \omega_{1}(c)\right],
$$

where $m_{z}$ is the out-of-plane carrier effective mass of the well material, $\gamma_{n}$ and $\omega_{n}$ with $n$ as an integer are simple functions of a variable defined by Eqs.(19). Next, the average potential due to charged impurities can be written in terms of the dimensionless doping positions: $d=z_{d} / L$ and $s=z_{s} / L$, by

$$
\left\langle V_{I}\right\rangle=\frac{4 p e^{2}}{e_{L}} \frac{N_{I} L^{2}}{2}\left(d^{2}-s^{2}\right) .
$$

Lastly, for the carrier potential it holds

$$
\begin{aligned}
\left\langle V_{s}\right\rangle= & \frac{4 \pi e^{2}}{\varepsilon_{L}} \frac{p_{s} L}{4}\left\{\pi B^{2}\left[\left(2 \frac{g_{-}}{L}+g_{-}^{\prime}\right) \gamma_{l}(c)-g_{+}^{\prime} \frac{\partial \gamma_{l}(c)}{\partial c}\right]\right. \\
& -\frac{\pi^{2} B^{4}}{4} \gamma_{I}(2 c)\left[\frac{1}{c^{2}}+\frac{c^{2}-\pi^{2}}{\left(c^{2}+\pi^{2}\right)^{2}}+\frac{c^{2}-\pi^{2}}{2\left(c^{2}+\pi^{2}\right)^{2}}\left[\gamma_{2}(2 c)-\gamma_{0}(2 c)\right]\right] \\
& \left.+\frac{\pi^{2} B^{4}}{4}\left[\frac{\pi c}{\left(c^{2}+\pi^{2}\right)^{2}}\left[\omega_{2}(2 c)+2 \omega_{I}(2 c)\right]\right]\right\}
\end{aligned}
$$

\section{Low-temperature quantum transport}

We are now dealing with quantum transport of electrons in the above system. The particles moving in the in-plane are scattered by various disorder sources, which are characterized by some random fields. Acoustic-phonon scattering has the momentum relaxation time given by [6] 


$$
\frac{1}{\tau(E)}=\sum_{k^{\prime}} W\left(k, k^{\prime}\right)(1-\cos \alpha)
$$

where $k=k_{x} \hat{i}+k_{y} \hat{j}$ and $W\left(k, k^{\prime}\right)$ is the transition probability from the state $k$ to $k^{\prime}$ and $\alpha$ is the scattering angle.

As known, $[6,7]$ scattering by a Gaussian random field is the transition probability $W\left(k, k^{\prime}\right)$ :

$$
W\left(k, k^{\prime}\right)=\frac{2 k_{B} T}{\hbar^{2}} \int_{-\infty}^{+\infty} d z \frac{|M(q)|^{2}}{\omega_{q} S^{2}\left(\left|k-k^{\prime}\right|\right)}\left|I\left(q_{z}\right)\right|^{2} \delta\left(E_{k}-E_{k^{\prime}}\right)
$$

where $q_{z}$ is the wave vector normal to the heterointerface and $|M(q)|$ is the 3D scattering matrix element.

$I\left(q_{z}\right)$ is the overlap integral by [6]

$$
I\left(q_{z}\right)=\int_{-\infty}^{+\infty} d z \zeta^{*}(z) \exp \left(i q_{z} z\right) \zeta(z) \text {. }
$$

At high temperatures the carriers in the single-side doped square QWs are expected to experience the following scattering sources: acoustic phonons (Deformation potential-DP(i) and Piezoelectric coupling-PZ(ii)).

Total inverse transport lifetime:

$$
\frac{1}{\tau_{t o t(A C)}}=\frac{1}{\tau_{D P}}+\frac{1}{\tau_{P Z}}
$$

Within the linear transport theory the mobility is determined by $\mu=e \tau_{t} / m^{*}$, with $m^{*}$ as the inplane carrier effective mass of the well layer.

(i). Deformation potential-DP:

For the acoustic phonons generated by the deformation potential the electron-phonon scattering matrix element is given by: [7]

$$
|M(q)|^{2}=\frac{D^{2} \hbar \omega_{q}}{2 c_{l} L^{3}}
$$

where $D$ is the deformation potential constant.

\section{(ii). Piezoelectric coupling-PZ}

Scattering matrix element due to the piezoelectric coupling given by [7]

$$
|M(q)|^{2}=\frac{2 \pi e^{2} P^{2} \hbar \omega_{q}}{\epsilon_{0} q^{2} L^{3}}
$$

where $P$ is the piezoelectric constant, ${ }^{\epsilon_{0}}$ is the static dielectric constant, ${ }^{e}$ the elemental charge.

The static screening factor at finite temperature has been calculated to be $[8,9]$ 


$$
S(q)=1+\frac{2 \pi e^{2} F(q) \Pi(q)}{\epsilon_{0} q}
$$

The screening form factor $F_{s}(q)$ takes account of the extension of the particle state along the growth direction, defined by [8]

$$
F_{S}(q)=\int_{-\infty}^{+\infty} d z \int_{-\infty}^{+\infty} d z^{\prime} \zeta^{2}(z) \zeta^{2}\left(z^{\prime}\right) e^{-q\left|z-z^{\prime}\right|}
$$

The calculation of $F_{s}(q)$ with plausible wave function given in Eq. (1) is lengthy, but straight forward with the aid of the functions $\gamma_{n}$ and $\omega_{n}$ in Eqs:

$$
\begin{aligned}
& \gamma_{n}(x)=\left[\frac{1}{x}+\frac{(-1)^{n} x}{x^{2}+n^{2} \pi^{2}}\right] \sinh x, \\
& \omega_{n}(x)=\frac{(-1)^{n} n \pi}{x^{2}+n^{2} \pi^{2}} \sinh x .
\end{aligned}
$$

As a result, one may achieve an analytic expression:

$$
F_{S}(t)=\left(\pi^{2} B^{4} / 4\right)\left[S^{(u)}(t)+S^{(l)}(t)\right] \text {, }
$$

with $t=q L$ as the dimensionless in-plane wave number. The functions figuring here are defined as follows:

$$
\begin{aligned}
& S^{(u / l)}(t)= \pm \frac{1}{2(c \mp t / 2)}\left[e^{ \pm(c \mp t / 2)} \gamma_{l}(c \pm t / 2)-\gamma_{l}(2 c)\right] \pm \frac{\pi}{4\left[\pi^{2}+(c \mp t / 2)^{2}\right]}\left[\omega_{2}(2 c)\right. \\
& \left.+2 \omega_{I}(2 c)\right] \mp \frac{c \mp t / 2}{4\left[\pi^{2}+(c \mp t / 2)^{2}\right]}\left[2 e^{ \pm(c \mp t / 2)} \gamma_{l}(c \pm t / 2)+\gamma_{2}(2 c)+2 \gamma_{l}(2 c)-\gamma_{0}(2 c)\right],
\end{aligned}
$$

where the upper and lower signs refer to the superindices $(u)$ and $(l)$, respectively.

The static polarizability at finite temperature is given by [7]

$$
\Pi\left(q, T, E_{F}\right)=\frac{1}{4 k_{B} T} \int_{0}^{+\infty} \frac{\Pi(q, 0, \sigma)}{\cosh ^{2}\left(\frac{E_{F}-\sigma}{2 k_{B} T}\right)} d \sigma,
$$

where $\Pi(q, O, \sigma)$ is the polarizability function at $T=O \mathrm{~K}$, namely,

$$
\Pi(q, 0, \sigma)=\frac{m^{*}}{\pi \hbar^{2}}\left\{1-\theta\left(q-2 k_{F}\right)\left(1-\left(\frac{2 k_{F}}{q}\right)^{2}\right)^{1 / 2}\right\},
$$

here $k_{F}=\sqrt{2 m^{*} E_{F}} / \hbar$ is the Fermi wave number. In the quantum limit, the temperature can be considered low enough so that $q<2 k_{F}$ becomes a very good approximation to acoustic phonon and it is very useful in phonon mobility calculations since it makes the factor in brackets of Eq. (23) to be 
unity. Also, due to the fact that significant effects of interface-phonons were never experimentally found, the electron-phonons non interactions occurring in the 2DEG are considered identical to that of bulk GaAs.

\section{Numerical results}

In this section, we apply the above-developed theory in order to understand the properties of lowtemperature transport in remote doped square QWs. The calculations of electron mobility were developed for $G a A s / A l_{x} G a_{l-x} A s$ square QWs whose following physical parameters were taken elsewhere $[7,10]$ :

There common parameters are: $L=152 \AA, p_{s}=10^{12} \mathrm{~cm}^{-2}, L_{s}=200 \AA, L_{d}=100 \AA, \varepsilon_{L}=13.4, x=0,33$, $a_{B}^{*}=103.47 \AA, C_{l}=1.397 \times 10^{11} \mathrm{~N} / \mathrm{m}^{2} \mathrm{~m}^{*}=0.067 \mathrm{~m}_{e}, P=0.064$

These mobility dependences on temperature for square quantum well are shown in Figure 1. Acoustic-phonon partial mobility in a $G a A s / A l_{x} G a_{l-x} A s$ square QW with a chanel width vs temperature $\mathrm{T}$. The experimental data [10] are marked by squares.
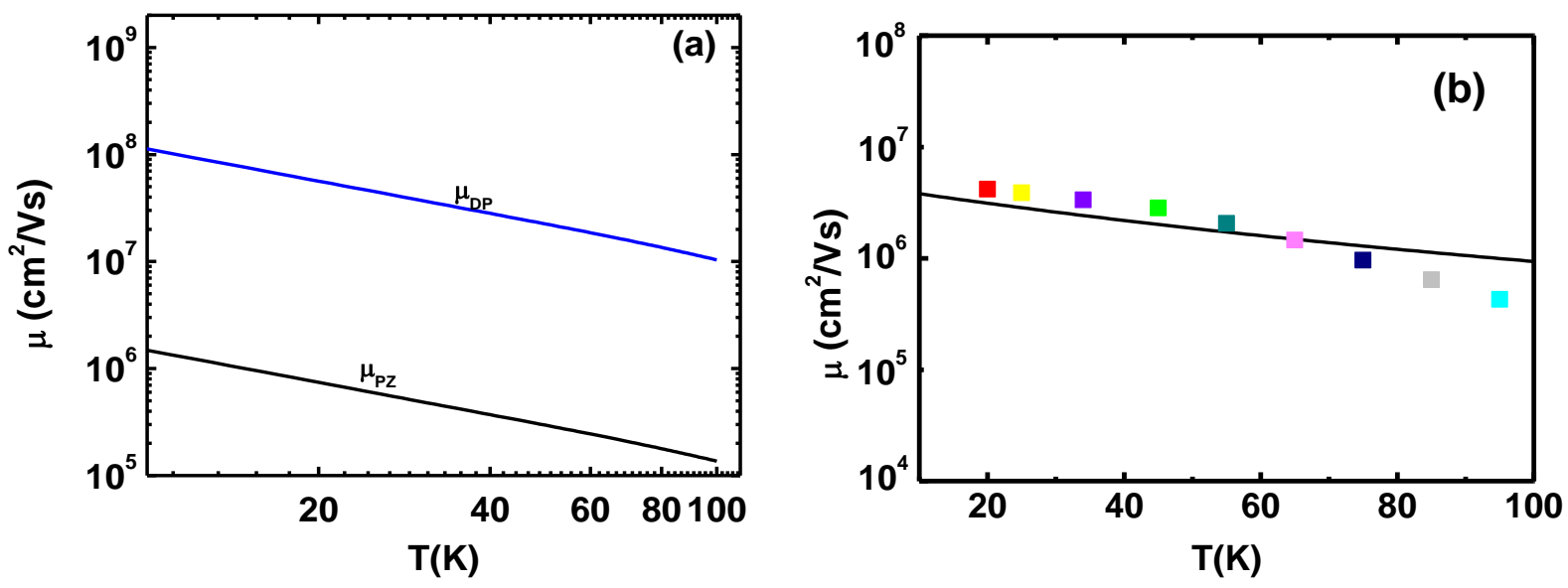

Acoustic-phonon (DP plus PZ coupling) partial mobility dependence on temperature for singleside doped square quantum well.

As clearly observed from Figure $1(a, b)$, the partial mobility for Deformation potential-DP and Piezoelectric coupling-PZ (a) and the total mobility (b) decreased in the temperature values $1 \div 100 \mathrm{~K}$.

\section{Conclusion}

(i) Within the variational approach, we obtain analytic expressions for the carrier distribution and their scattering in single-side modulation-doped square quantum wells.

(ii) We calculated the dependences on temperature of acoustic-phonon scattering and the total mobility. We show that phonon scattering plays an important role in limiting the electron mobility in square QWs. We analyzed the behavior of the mobility for temperature values up to $100 \mathrm{~K}$. 
(iii) Our theory is able to well reproduce the recent experimental data about transport of electrons and holes in one-side doped square QWs.

\section{Acknowledgments}

The authors are grateful to supports from Nguyen Suan Han, Nguyen Quang Bau, Faculty of Physics, University of Science, Vietnam National University, Hanoi, Vietnam.

\section{References}

[1] F. Schaffler, Semicond. Sci. Technol. 12, 1515 (1997).

[2] T. E. Whall and E. H. C. Parker, J. of Phys. D 31, 1397 (1998).

[3] D. N. Quang and N. H. Tung, Phys. Rev. B, 77 (2008) 125335.

[4] D. N. Quang, N. H. Tung, D. T. Hien, and T. T. Hai, J. Appl. Phys., 104 (2008) 113711.

[5] A. Gold, Phys. Rev. B, 35 (1987) 723; 38 (1988) 10798.

[6] C. Weisbuch and B. Vinter, Quantum Semiconductor Structures, Academic Press, Orsay (France) 1991.

[7] F. M. S. Lima, Qu Fanyao, O. A. C. Nunes, and A. L. A. Fonseca, phys. stat.sol (b), No. 1, (2001) 43-61.

[8] Y. Okuyama and N. Tokuda, Phys. Rev. B 40, 9744 (1989).

[9] F. Stern, Phys. Rev. Lett. 18, 546 (1967).

[10] V. Umansky, R. De-Picciotto, and M. Heiblum, Appl. Phys. Lett, 39 (1981) 912. 